\title{
Cardamom Oil
}

National Cancer Institute

\section{Source}

National Cancer Institute. Cardamom Oil. NCI Thesaurus. Code C107279.

The essential oil from Elettaria cardomomum. Cardamom oil is used for its aromatic properties, in cooking, and in medicinal preparations. 